GUEST EDITORIAL

\title{
Tattoos: Their changing demographics require clinicians to be more knowledgeable about their impact on health
}

Fuelled by internet-driven global connectedness, social media, television shows such as Miami Ink and the quest for individuality, a growing proportion of the global population is acquiring tattoos. As tattooing is increasingly being perceived as an art form, more celebrities are obtaining tattoos and tattoo artists are becoming celebrities in their own right. Consequently, the demographics of people with tattoos are gradually changing. Tattoos are no longer the domain of sailors, gangsters and motorcyclists, but are being embraced by wider cross-sections of society. ${ }^{[1-3]}$ In 2016, it was estimated that $14 \%$ of the US population had tattoos, rising to $40 \%$ among those aged 26 - 40 years. However, $17 \%$ of tattooed individuals regretted having a tattoo and $11 \%$ were either having it removed or already had it removed. ${ }^{[4]}$ Unwanted tattoos pose obvious problems, such as financial costs of professional removal, incomplete removal and residual scarring. Other challenges of tattooed persons are often overlooked, and are inadequately or inappropriately managed by healthcare providers owing to lack of sufficient knowledge. These challenges include psychological sequelae, acute and chronic tattoo complications and effects of tattoos on diagnostic and therapeutic medical procedures. Another under-recognised problem is the need to help de-label and reintegrate reformed prisoners and gang members into society. ${ }^{[5]}$ This requires more attention than it currently receives in South Africa (SA). Cosmetic tattooing after medical procedures such as a mastectomy is also underutilised.

Studies from 20 years ago suggest that healthcare practitioners had negative perceptions of tattooed persons seeking care. ${ }^{[6]}$ However, prejudices that have historically been associated with tattoos are diminishing globally, as tattoos weave themselves into the popular culture. The previous and current effects of these prejudices on patient care and outcomes are unclear. As the prevalence of tattoos increases, it is inevitable that expertise will more often be sought from healthcare providers who may not be adequately informed. We hope that the CME article ${ }^{[7]}$ will provide clinicians with a basic knowledge and understanding of tattoos to guide them in the management of those seeking tattoo-related advice, or be able to point them in the right direction. The article briefly summarises a wide range of topics on tattoos, ranging from classification, epidemiology, motivations to obtain a tattoo, process of tattooing, medical applications, complications, reasons for and methods of removal and a summary of the current legislative framework in SA.
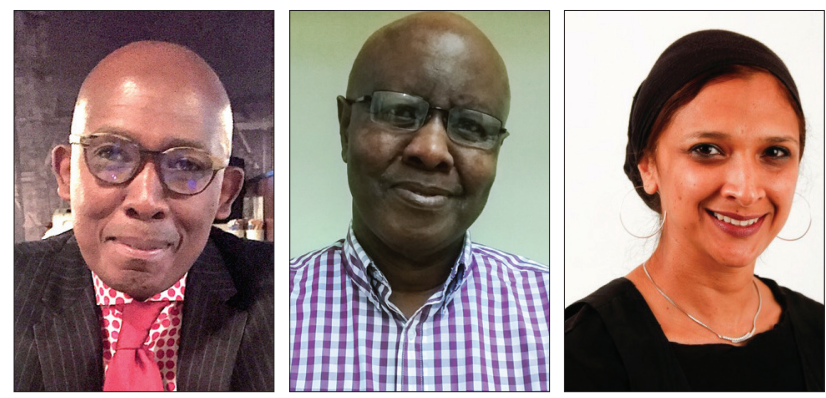

Rannakoe J Lehloenya, Reginald M Ngwanya, Thuraya Isaacs Division of Dermatology, Department of Medicine, Faculty of Health Sciences, Groote Schuur Hospital and University of Cape Town, South Africa

rannakoe.lehloenya@uct.ac.za

1. Muller CS, Oertel A, Korner R, Pfohler C, Vogt T. Socio-epidemiologic aspects and cutaneous side effects of permanent tattoos in Germany - tattoos are not restricted to a specific social phenotype. Dermatoendocrinol 2017;9(1):e1267080. https://doi.org/10.1080/19381980.2016.1267080

2. Camacho JN. The Tattoo: A Mark of Subversion, Deviance, or Mainstream Self-expression? Florida, USA: University of South Florida, 2014.

3. Velliquette AM, Murray JB, Creyer EH. The tattoo Renaissance: An ethnographic account of symbolic consumer behavior. Adv Consume Res 1998;25:461-467.

Tattoo Statistics. Statistic Brain Research Institute. 2017. https://www.statisticbrain.com/tattoo-statistics (accessed 26 March 2018)

Poljac B, Burke T. Erasing the past: Tattoo-removal programs for former gang members. FBI Law Enforcement Bull 2008; $77(8): 13-18$

6. Stuppy DJ, Armstrong ML, Casals-Ariet C. Attitudes of health care providers and students towards tattooed people. J Adv Nurs 1998;27(6):1165-1170. https://doi.org/10.1046/j.1365-2648.1998.00626.x

Lehloenya RJ, Ngwanya RM, Isaacs T. Tattoos: A summary knowledge for the practising clinician

S Afr Med J 2018;108(9):714-720. https://doi.org/10.7196/SAMJ.2018.v108i9.13231

S Afr Med J 2018;108(9):713. DOI:10.7196/SAMJ.2018.v10819.13303 